\title{
Distribuição temporal e espacial de anuros em área de Pampa, Santa Maria, RS
}

\author{
Tiago Gomes dos Santos ${ }^{1}$, Katia Kopp ${ }^{2}$, Marcia Regina Spies $^{3}$, Rafael Trevisan ${ }^{4}$ \\ $\&$ Sonia Zanini Cechin ${ }^{5}$
}

1. Programa de Pós-Graduação em Ciências Biológicas (Zoologia), IB, Universidade Estadual Paulista, Av. 24A, n 1515 , Bairro Bela Vista, 13506-900 Rio Claro, SP, Brasil. (frogomes@yahoo.com.br)

2. Programa de Pós-Graduação em Ciências Ambientais- CIAMB, ICB 4 - Secretaria de Pós-Graduação em Ciências Ambientais, Campus Samambaia, Universidade Federal de Goiás, Caixa Postal: 131, 74001-970 Goiânia, GO, Brasil. (kakopp@ hotmail.com)

3. Programa de Pós-Graduação em Entomologia, Faculdade de Filosofia Ciências e Letras de Ribeirão Preto, Universidade de São Paulo, Av. Bandeirantes, 3900, 14040-901 Ribeirão Preto, SP, Brasil. (marciaspies@gmail.com)

4. Programa de Pós-Graduação em Botânica, Universidade Federal do Rio Grande do Sul, Av. Bento Gonçalves, 9500, prédio 43433, sala 214, 91501-970 Porto Alegre, RS, Brasil. (rftrevisan@yahoo.com.br)

5. Departamento de Biologia, Centro de Ciências Naturais e Exatas, Universidade Federal de Santa Maria, Av. Roraima s/ nº $97105-900$ Santa Maria, RS, Brasil. (cechinsz@ccne.ufsm.br)

\begin{abstract}
Temporal and spatial distribution of anurans in the Pampa Region (Santa Maria, RS). The present study aimed to determine the richness, occurrence constancy, reproductive modes, standard of abundance distribution, season of vocalization and to test correlation among climatic variables and activity of vocalization of anurans in a region of the Pampa Biome, Santa Maria, Rio Grande do Sul State. During the period of November/2001 to October/2002 monthly collections were carried out utilizing the 'survey at breeding site' method and examination of specimens kept in the Coleção Herpetológica do Setor de Zoologia da Universidade Federal de Santa Maria (ZUFSM). The occurrence of 25 species of anurans was recorded. The anurofauna recorded represents $30 \%$ of the species known to occur in Rio Grande do Sul, and comprises species generally associated with grasslands in this state and neighboring countries. Four reproductive modes were recorded: mode 1 (14 species; 58.3\%); mode 11 and 30 (9 species; $37.5 \%$ ) and mode 24 (1 species; $4.2 \%$ ). The low diversification of reproductive modes is likely related to the homogeneity of the grassland habitat. Most species were constant or accessory in the study area and the species abundance distribution patterns fit in the Broken Stick and Log-normal models, characterized by homogeneity of species abundance distribution. Most species showed great plasticity in habitat, but few were plastic in vocalization sites use. There was a weak positive correlation between species richness and precipitation. There was also a weak positive correlation between the abundance of species calling activity and maximum average temperatures. These correlations indicated that, in the study area, the abundance of calling males is more affected by the temperature, and species richness is more affected by precipitation, despite the fact that significantly higher species richness occurs during the hottest period of the year. These results showed that the climatological variables examined were not enough to explain the seasonal occurrence of species, thus the influence of other environmental variables merit to be tested in future studies.
\end{abstract}

KEYWORDS. Anuran community, abundance models, reproductive modes, seasonality, subtropical region.

RESUMO. Este estudo teve como objetivo determinar a riqueza, a constância de ocorrência, os modos reprodutivos, o padrão de distribuição da abundância, a temporada de vocalização e testar a correlação das variáveis climáticas sobre a atividade de vocalização dos anuros em uma região do Bioma Pampa, Santa Maria, Rio Grande do Sul. Durante o período de novembro de 2001 a outubro de 2002 foram realizadas coletas mensais empregando o método de 'busca em sítio de reprodução' e exame de exemplares depositados na Coleção Herpetológica do Setor de Zoologia da Universidade Federal de Santa Maria (ZUFSM). Foi registrada a ocorrência de 25 espécies de anuros. A anurofauna registrada corresponde a $30 \%$ das espécies encontradas no Rio Grande do Sul e normalmente está associada a áreas abertas encontradas no estado e em países vizinhos. Foram registrados quatro modos reprodutivos: modo 1 (14 espécies; 58,3\%); modos 11 e 30 (nove espécies; 37,5\%) e modo 24 (uma espécie; 4,2\%). A baixa diversificação de modos reprodutivos provavelmente está relacionada à homogeneidade do hábitat primariamente campestre. A maior parte das espécies mostrou-se constante ou acessória na área estudada e o padrão de distribuição da abundância das espécies apresentou ajuste aos modelos Broken Stick e Log-normal, caracterizados pela homogeneidade na distribuição da abundância das espécies. A maioria das espécies apresentou grande plasticidade na ocupação de hábitats, mas poucas foram plásticas no uso dos sítios de vocalização. Houve correlação positiva, ainda que fraca, da riqueza de espécies com a precipitação mensal acumulada e da abundância com a temperatura média máxima. As correlações obtidas indicaram que na área estudada a temperatura parece atuar mais sobre a abundância de machos em atividade de vocalização e a precipitação sobre a riqueza, apesar da riqueza de espécies ser significativamente maior durante o período mais quente do ano. Estes resultados revelaram que as variáveis climatológicas testadas explicaram muito pouco da ocorrência sazonal das espécies, assim a influência de outras variáveis ambientais merece ser testada em estudos futuros.

PALAVRAS-CHAVE. Comunidade de anuros, modelos de abundância, modos reprodutivos, sazonalidade, região subtropical.

Estudos sobre ecologia e história natural de anuros no Brasil são de grande importância para propósitos de conservação (ETERovick et al., 2005), porém escassos frente à grande riqueza de espécies do país (804 espécies, SBH 2008). No estado do Rio Grande do Sul a falta de conhecimento sobre as espécies ocorrentes é um fator limitante na avaliação da situação de conservação dos anfíbios anuros (MMA, 2002), já que os estudos existentes são extremamente fragmentários, basicamente de cunho taxonômico (GARCIA \& Vinciprova, 2003) e enfocam listas ou descrições e ampliações de distribuição de espécies (BRAUn \& BrAUn, 1974, 1976, 1980; GAYER et al., 1988; Garcia \& Vinciprova, 1999; Kwet, 2000; Kwet 
\& Di-Bernardo, 1998; Kwet \& Flavovich, 2001; Cechin et al., 2002; Kopp et al., 2002; LoEBMANN \& VieIRA, 2005).

Os poucos e recentes estudos abordando aspectos ecológicos da anurofauna do Rio Grande do Sul têm sido desenvolvidos na metade norte do estado, tanto em áreas florestadas (Floresta Ombrófila Mista) quanto campestres, pertencentes ao Planalto Meridional Brasileiro (Kwet \& Di-Bernardo, 1999; Kwet, 2001; GRANDo et al., 2004), enquanto estudos na metade sul, no Bioma Pampa, são quase inexistentes (Di-BERNARDO \& Kwet, 2002; Di-Bernardo et al., 2004). Assim, questões básicas como padrões de atividade temporal das espécies permanecem pouco exploradas. De acordo com DIBERNARDo et al. (2004), a maior atividade de vocalização dos anuros no extremo sul do Rio Grande do Sul (Candiota) foi registrada no período quente do ano (primavera e verão), como tipicamente ocorre em regiões de clima temperado, mas nenhuma correlação foi estatisticamente testada.

De forma geral, é esperado que a atividade reprodutiva dos anuros em regiões tropicais sazonais seja concentrada durante a estação quente e chuvosa do ano (e.g., Rossa-Feres \& Jim, 1994; Bertoluci \& Rodrigues, 2002a; Kopp \& Eterovick, 2006; SAntos et al., 2007), enquanto que em regiões tropicais não sazonais, muitas espécies apresentem atividade reprodutiva ao longo de todo ano (Duelman \& Trueb, 1986; Crump, 1974). Entretanto, para regiões subtropicais os resultados não confirmam um único padrão, já que alguns estudos apontam forte correlação das atividades reprodutivas dos anuros com o período quente e chuvoso do ano (CONTE \& MACHADO, 2005; Conte \& Rossa-Feres, 2006), enquanto outros apenas com o período mais quente ou ainda nenhuma correlação da atividade reprodutiva com a chuva ou temperatura (Bernarde \& MACHADO, 2001; BERNARDE \& ANJOS, 1999, respectivamente).

Dados coletados entre 1970-1980 mostram que $180.000 \mathrm{~km}^{2}$ da região sul do Brasil eram ocupados por campos (Leite \& KLEIN, 1990) e, apesar do estado atual de conservação do Bioma Pampa ser pouco conhecido, devido à falta de tecnologia capaz de diferenciar o uso da terra em regiões de vegetação campestre (MANTOVANi \& Silva, 2002), considera-se que os campos úmidos dos países ditos 'em desenvolvimento' estão ameaçados por pastoreio excessivo, queimadas, invasão de espécies exóticas e conversão em áreas agriculturáveis, restando muitas vezes apenas pequenos remanescentes em uma paisagem predominantemente agrícola (RISSER, 1997; Porto, 2002; Bencke, 2003). Além disso, os campos nativos, apesar de sustentarem importantes estoques da diversidade genética, têm sido menosprezados devido à baixa taxa de endemismos (RISSER, 1997), o que resulta em poucas áreas desta fitofisionomia efetivamente protegidas em Unidades de Conservação (MANTOVAnI \& Silva, 2002). Desta forma, considerando a carência do conhecimento sobre a herpetofauna de áreas campestres (HADDAD \& ABE, 2000) e o grau de ameaça dessa fitofisionomia, torna-se urgente o desenvolvimento de estudos visando caracterizar a estrutura e/ou organização das comunidades. Neste sentido, o desenvolvimento de estudos em áreas campestres alteradas é tão importante quanto estudos em áreas preservadas, pois subsidia dados necessários para futuras comparações entre áreas em diferentes estados de conservação, possibilitando a compreensão dos possíveis efeitos da degradação ambiental sobre as comunidades dessa fitofisionomia.

O presente estudo teve como objetivos: i) determinar a riqueza, a constância de ocorrência e a diversidade de modos reprodutivos da anurofauna em uma área de campo (Pampa) com grande pressão antrópica; ii) analisar o padrão de distribuição da abundância das espécies; iii) estabelecer a temporada de vocalização dos machos de cada espécie e iv) testar a correlação entre as variáveis climáticas de temperatura e precipitação com a riqueza e abundância de espécies em atividade de vocalização.

\section{MATERIAL E MÉTODOS}

Área estudada. O estudo foi desenvolvido no Campus da Universidade Federal de Santa Maria (UFSM), situado na Depressão Central do Rio Grande do Sul (29 $42^{\prime}$ S, 53 $42^{\circ}$ ' W; 95m de altitude) no bioma Pampa (IBGE, 2004). Aárea estudada possui cerca de $6 \mathrm{~km}^{2}$, sendo $35 \%$ urbanizados e o restante está irregularmente distribuído em uma paisagem bastante fragmentada, composta aproximadamente $30 \%$ de campo nativo com presença de gado bovino e ovino, $20 \%$ de lavouras (e.g. soja, milho, sorgo, horticultura, pastagens) e $15 \%$ restantes ocupados por capoeira, fragmentos de silvicultura de espécies exóticas (Eucalyptus e Pinus) e fragmentos de mata secundária em regeneração, com predomínio de Prunus sellowii Koehne (Rosaceae) (SANTos et al., 2005). De acordo com a classificação de Köppen, o município de Santa Maria apresenta clima subtropical úmido com verões rigorosos (Cfa), caracterizado por chuvas bem distribuídas ao longo do ano (índices pluviométricos entre 1500 e $1750 \mathrm{~mm}$ ) e médias térmicas entre $17^{\circ} \mathrm{C}$ e $19^{\circ} \mathrm{C}$ (PEREIRA et al., 1989).

Coleta de dados. As amostragens de campo foram realizadas mensalmente e tiveram duração de quatro dias/ mês, no período de novembro de 2001 a outubro de 2002. As atividades tiveram início ao anoitecer e se estenderam até por volta das 24:00h. Durante este período, o inventário e a estimativa de abundância de cada espécie (número de machos em vocalização) foram realizados pelo método de "busca em sítios de reprodução" (ScOTT \& WoODWARD, 1994), percorrendo-se o perímetro de 42 corpos d'água presentes nas diversas fisionomias da área estudada: cinco córregos permanentes, 16 banhados (nove permanentes e sete temporários), nove açudes permanentes e 12 poças (10 permanentes e duas temporárias). Nesse percurso, os seguintes dados foram registrados para os machos em atividade de vocalização: sítio de vocalização (solo, água ou empoleirado na vegetação), tipo de corpo d'água utilizado (banhado, córrego, açude ou poça) e tipo de matriz onde se encontravam os corpos d'água (área urbana, lavoura, campo, capoeira, mata). O esforço amostral em cada corpo d'água foi padronizado pelo tamanho e complexidade dos mesmos (SCOTT \& WoodWARd, 1994), ou seja, corpos d'água maiores e mais complexos foram investigados por maior período de tempo. 
Os modos reprodutivos das espécies foram classificados de acordo com a literatura (sensu HADDAD \& Prado, 2005) e observações no campo. Os dados climatológicos (temperatura média máxima e mínima mensal e precipitação mensal acumulada) foram obtidos na Estação Meteorológica do Departamento de Fitotecnia, UFSM.

Espécimes-testemunho foram tombados na Coleção Herpetológica do Setor de Zoologia da Universidade Federal de Santa Maria (ZUFSM).

Análise estatística. A avaliação da eficiência de coleta foi realizada pela construção da curva do coletor, a partir de 100 adições aleatórias das amostras, utilizando o programa EstimateS 7.0 (Colwell, 2004).

A constância de ocorrência das espécies foi calculada pelo índice de SiLVEIRA-Neto et al. (1976), que considera constantes as espécies encontradas em mais de $50 \%$ das amostras; acessórias aquelas presentes entre 25 e $50 \%$ e ocasionais as espécies que foram encontradas em menos de $25 \%$ das amostras.

O padrão de distribuição da abundância das espécies foi analisado pelo teste de ajuste (qui-quadrado) a quatro modelos de abundância (Broken Stick, Lognormal, Séries Geométrica e Logarítmica; ver referências em Magurran, 1988), utilizando o programa Bio-Dap (MagurRan, 1988). Para esta análise, a abundância total de cada espécie foi considerada como igual a do mês com maior abundância (BERTOLUCI \& RoDRIGUES, 2002a, VAsconcelos \& Rossa-Feres, 2005) ao longo das 12 amostragens. Esse procedimento foi adotado para evitar a super estimativa de abundância, promovida pela recontagem dos indivíduos em amostragens repetidas ao longo do período estudado.

A similaridade na distribuição temporal dos machos em atividade de vocalização foi analisada pelo Índice de Similaridade Jaccard (Cj), com posterior análise de agrupamento pelo método da média não ponderada (UPGMA) (KREBS, 1999), utilizando o programa NTSYS 2.1 (Rohlf, 2000). Foram considerados como agrupamentos válidos aqueles com similaridade superior a $50 \%$. A perda de informações na construção dos dendrogramas foi avaliada pelo Coeficiente de Correlação Cofenética (r) (Romesburg, 1984), obtido da correlação da matriz de similaridade original com a matriz obtida a partir do dendrograma, sendo valor de $r \geq 0,9$ considerado um ajuste muito bom; $0,8 \leq \mathrm{r}<0,9$ um ajuste bom, $0,7 \leq \mathrm{r}$ $<0,8$ um ajuste pobre e $\mathrm{r}<0,7$ um ajuste muito pobre entre as matrizes (RoHLF, 2000).

O teste de Mann-Whitney (U) foi utilizado para comparar a riqueza de espécies (considerando esta como o número de espécies com machos em atividade de vocalização) e os dados climatológicos entre os períodos de menor e maior riqueza (ZAR, 1999). As análises foram realizadas no programa BioEstat 3.0 (AYREs et al., 2003).

A correlação da riqueza e da abundância de espécies em atividade de vocalização com as variáveis climatológicas de temperatura média mínima (Tmin), temperatura média máxima (Tmax) e precipitação mensal acumulada $(\mathrm{Pa})$ foi testada utilizando o teste de Mantel (Manly, 1994). Esse teste faz correlações de matrizes, utilizando a estatística $\mathrm{Z}$, onde o valor de $\mathrm{Z}$ depende do número e da magnitude de elementos nas matrizes a serem comparadas, assim é feita uma normalização que transforma o $\mathrm{Z}$ em um coeficiente $(\mathrm{r})$ que varia entre $+1 \mathrm{e}$ -1 . O teste de permutação de Monte Carlo foi utilizado para estabelecer a significância de Z (SMOUSE et al., 1986), com 5000 permutações. Para o teste de Mantel foram construídas matrizes de dissimilaridade (Distância Euclidiana) de cada variável climática e, matrizes de similaridade para os dados de riqueza (Jaccard) e abundância (Morisita-Horn) de espécies em atividade de vocalização (ver detalhes sobre os índices utilizados em KREBS, 1999). Antes de se proceder à análise principal, o teste de Mantel foi utilizado para verificar se meses mais próximos possuíam estrutura mais similar, aqui chamada de Coesão Temporal, quanto às variáveis climatológicas e quanto aos dados bióticos. Desta forma, as matrizes de dissimilaridade e similaridade foram tentativamente correlacionadas como uma matriz-hipótese de coesão temporal entre os meses, utilizando a distância temporal. Esta última matriz de dissimilaridade foi construída em graus, considerando a menor distância em um ciclo anual, percorrendo-se o ciclo no sentido horário e anti-horário. Desta forma, a distância entre novembro e fevereiro foi considerada $90^{\circ}$, e entre novembro e junho foi considerada $150^{\circ}$ e não $210^{\circ}$. Em caso de existência de coesão temporal nos conjuntos de dados, as análises de correlações foram posteriormente realizadas pelo teste de Mantel Parcial (SMouse et al., 1986), para retirar o efeito temporal na estrutura dos dados sobre o resultado das correlações. Este método consiste em comparar duas matrizes (A e B) retirando o efeito de uma terceira matriz $(\mathrm{C})$ sobre elas, utilizando uma regressão de $\mathrm{C}$ sobre $\mathrm{A}$ e sobre $\mathrm{B}$, obtendo duas matrizes de resíduos que representam a parte da variação de A e de B que não é explicada por C (SMOusE et al., 1986). Assim, as duas matrizes de resíduos podem ser compradas normalmente. A construção das matrizes e os testes de Mantel e Mantel Parcial foram realizados pelo programa NTSYS 2.1 (RoHLF, 2000).

\section{RESULTADOS E DISCUSSÃO}

Riqueza, constância de ocorrência, modos reprodutivos e padrão de abundância.

Durante as atividades de campo foram registradas 24 espécies de anuros e o exame de espécimes tombados na Coleção Herpetológica do Setor de Zoologia da Universidade Federal de Santa Maria (ZUFSM) permitiu a inclusão de mais um hilídeo (Phyllomedusa iheringii Boulenger, 1885) à listagem, totalizando 25 espécies distribuídas em 12 gêneros de sete famílias: Bufonidae (1), Cycloramphidae (1), Hylidae (11), Leiuperidae (5), Leptodactylidae (5), Microhylidae (1) e Ranidae (1) (Tab. I).

A curva média do coletor, apesar de apresentar tendência de estabilização, ainda possui uma pequena variação ao final das amostragens (Fig. 1) e, desta forma um incremento na riqueza de espécies não pode ser descartado com a continuidade do esforço de coleta. $\mathrm{O}$ registro, na Coleção Herpetológica, de outras duas espécies de leptodactilídeos (Leptodactylus chaquensis Cei, 1950 e L. furnarius Sazima \& Bokermman, 1978) para áreas campestres próximas reforça a possibilidade de incremento de espécies na anurofauna da área estudada. 
As 24 espécies nativas registradas no presente estudo correspondem a $30 \%$ das 79 listadas para o Rio Grande do Sul (GARCIA \& Vinciprova, 2003) e a 77\% das 22 espécies de anuros registradas em área campestre na região de Candiota, RS (Di-BERNARDo et al., 2004). As espécies que compõem a anurofauna aqui estudada são comumente associadas a áreas abertas do estado e de países vizinhos, como Argentina e Uruguai (CEI, 1980; LANGONe, 1994; Cespedez et al., 1995; ACHAVAl \& Olmos, 1997; Kwet \& Di-Bernardo, 1998; Maneyro \& LANGONE, 2001; Alvarez et al., 2002). Todas as espécies já possuíam ocorrência conhecida para a região central do Rio Grande do Sul (Braun \& Braun, 1980; Garcia \& Vinciprova, 1999), exceto Hypsiboas albopunctatus (Spix, 1824) e Scinax nasicus (Cope, 1862) que constituíram novos registros para esta região do estado (CECHIN et al., 2002; Kopp et al., 2002). É importante destacar o encontro de Lithobates catesbeianus (Shawn, 1802) na área estudada; espécie exótica invasora, que nas últimas décadas tornouse abundante em várias regiões do país, após escapar de criatórios comerciais. Esta espécie atualmente ocupa quase todas as regiões do estado (MARTINS et al., 2002), em altas densidades, inclusive no interior de unidades de conservação (S.T.Z. Cechin, obs. pes.). Em um estudo realizado na região central do RS, $L$. catesbeianus predou nove das 24 espécies de anuros registrados no local (R. Boelter, com. pes.).

Das espécies registradas, doze foram consideradas constantes, sete acessórias e cinco ocasionais na área estudada (Tab. I). A maioria das espécies $(\mathrm{n}=17 ; 77 \%)$

Tabela I. Espécies de anuros registradas entre novembro de 2001 e outubro de 2002, em uma área de Pampa, Santa Maria, RS. FO, freqüência de ocorrência; IC, índice de constância (SilveIRA-Neto et al., 1976): C (espécies constantes), A (espécies acessórias) e O (espécies ocasionais); MR, modos reprodutivos (sensu Haddad \& Prado, 2005); *, registro de espécime depositado na Coleção Herpetológica da Instituição (ZUFSM). As barras cinzas indicam a ocorrência temporal de machos em atividade de vocalização.

\begin{tabular}{|c|c|c|c|c|c|c|c|c|c|c|c|c|c|c|c|}
\hline \multirow{2}{*}{ Familia / espécie } & \multirow{2}{*}{ FO (\%) } & \multirow{2}{*}{ IC } & \multirow{2}{*}{ MR } & \multicolumn{12}{|c|}{ Temporada de vocalização } \\
\hline & & & & $\mathrm{N}$ & $\mathrm{D}$ & $\mathrm{J}$ & $\mathrm{F}$ & M & A & $\mathrm{M}$ & $\mathrm{J}$ & $\mathbf{J}$ & A & $\mathrm{S}$ & $\mathrm{O}$ \\
\hline \multicolumn{16}{|l|}{ BUFONIDAE } \\
\hline Rhinella icterica (Spix, 1824) & 75 & $\mathrm{C}$ & 1 & & & & & & & & & & & & \\
\hline \multicolumn{16}{|l|}{ CYCLORAMPHIDAE } \\
\hline Odontophrymus americanus (Duméril \& Bibron, 1841) & 25 & A & 1 & & & & & & & & & & & & \\
\hline \multicolumn{16}{|l|}{ HYLIDAE } \\
\hline Dendropsophus minutus (Peters, 1872) & 83,33 & $\mathrm{C}$ & 1 & & & & & & & & & & & & \\
\hline Dendropsophus sanborni (Schmidt, 1944) & 75 & $\mathrm{C}$ & 1 & & & & & & & & & & & & \\
\hline Hypsiboas albopunctatus (Spix, 1824) & 8,33 & $\mathrm{O}$ & 1 & & & & & & & & & & & & \\
\hline Hypsiboas pulchellus (Duméril \& Bibron, 1841) & 83,33 & $\mathrm{C}$ & 1 & & & & & & & & & & & & \\
\hline Phyllomedusa iheringii Boulenger, $1885 *$ & - & - & 24 & & & & & & & & & & & & \\
\hline Pseudis minuta Günther, 1858 & 25 & A & 1 & & & & & & & & & & & & \\
\hline Scinax berthae (Barrio, 1962) & 66,67 & $\mathrm{C}$ & 1 & & & & & & & & & & & & \\
\hline Scinax fuscovarius (Lutz, 1925) & 50 & A & 1 & & & & & & & & & & & & \\
\hline Scinax granulatus (Gallardo, 1961) & 16,67 & $\mathrm{O}$ & 1 & & & & & & & & & & & & \\
\hline Scinax nasicus (Cope, 1862) & 16,67 & $\mathrm{O}$ & 1 & & & & & & & & & & & & \\
\hline Scinax squalirostris (Lutz, 1925) & 83,33 & $\mathrm{C}$ & 1 & & & & & & & & & & & & \\
\hline \multicolumn{16}{|l|}{ LEIUPERIDAE } \\
\hline Physalaemus biligonigerus (Cope, 1861) & 25 & A & 11 & & & & & & & & & & & & \\
\hline Physalaemus cuvieri Fitzinger, 1826 & 58,33 & $\mathrm{C}$ & 11 & & & & & & & & & & & & \\
\hline Physalaemus henselii (Peters, 1872) & 16,67 & $\mathrm{O}$ & 11 & & & & & & & & & & & & \\
\hline Physalaemus riograndensis Milstead, 1960 & 41,67 & A & 11 & & & & & & & & & & & & \\
\hline Pseudopaludicola falcipes (Hensel, 1867) & 83,33 & $\mathrm{C}$ & 1 & & & & & & & & & & & & \\
\hline \multicolumn{16}{|l|}{ LEPTODACTYLIDAE } \\
\hline Leptodactylus fuscus (Schneider, 1799) & 58,33 & $\mathrm{C}$ & 30 & & & & & & & & & & & & \\
\hline Leptodactylus gracilis (Duméril \& Bibron, 1841) & 66,67 & $\mathrm{C}$ & 30 & & & & & & & & & & & & \\
\hline Leptodactylus latinasus Jiménez de la Espada, 1875 & 16,67 & $\mathrm{O}$ & 30 & & & & & & & & & & & & \\
\hline Leptodactylus mystacimus (Burmeister, 1861) & 33,33 & A & 30 & & & & & & & & & & & & \\
\hline Leptodactylus cf. ocellatus (Linnaeus, 1758) & 91,67 & $\mathrm{C}$ & 11 & & & & & & & & & & & & \\
\hline \multicolumn{16}{|l|}{ MICROHYLIDAE } \\
\hline Elachistocleis bicolor (Guérin-Méneville, 1838) & 41,67 & A & 1 & & & & & & & & & & & & \\
\hline \multicolumn{16}{|l|}{ RANIDAE } \\
\hline Lithobates catesbeianus (Shawn, 1802) & 91,67 & $\mathrm{C}$ & 1 & & & & & & & & & & & & \\
\hline Riqueza de espécies & & & & 14 & 09 & 15 & 14 & 12 & 04 & 07 & 09 & 04 & 15 & 13 & 19 \\
\hline
\end{tabular}


apresentou grande plasticidade na ocupação de hábitats (tipos de corpos d'água e de matriz), mas poucas foram plásticas no uso dos sítios de vocalização $(\mathrm{n}=8 ; 34 \%)$ (Tab. II).

Especializações nos sítios de vocalização foram registradas em outros estudos (e.g., HöDL, 1977; Bertoluci \& Rodrigues, 2002b) e podem estar relacionadas aos modos reprodutivos, bem como a limitações morfológicas, fisiológicas ou comportamentais das espécies (CRUMP, 1971; CARDoso et al., 1989). A plasticidade no uso de hábitats registrada no presente estudo pode estar relacionada ao tipo de vegetação e também ao grau de perturbação antrópica da área, já que anfíbios anuros de áreas predominantemente abertas e/ ou alteradas pelo homem geralmente utilizam amplamente os recursos (Heyer \& Bellin, 1973; VAsconcelos \& Rossa-Feres, 2005; SANTOS et al., 2007).

Foram registrados quatro modos reprodutivos dentre as 24 espécies nativas ocorrentes na área estudada (Tab. I). O modo 1 (modo reprodutivo generalizado, caracterizado pela deposição de ovos e desenvolvimento de girinos em ambientes lênticos) representou a maior proporção de espécies (14 espécies; 58,3\% do total), seguido pelos modos 11 e 30 (desova em ninho de espuma depositado na superfície da água e em câmara subterrânea, respectivamente, com desenvolvimento dos girinos em ambientes lênticos: nove espécies; $37,5 \%$ ) e pelo modo 24 (uma espécie; 4,2\%), caracterizado por deposição de ovos em folhas de árvores e girinos que caem e se desenvolvem em ambientes lênticos (Tab. I).

PRADO et al. (2005), em estudo realizado no Pantanal brasileiro, considerando os padrões de atividade reprodutiva, modos reprodutivos e uso de hábitat por anuros, encontraram maior proporção de espécies com modo reprodutivo generalizado e, sugerem que a pronunciada estação seca e outros fatores como a homogeneidade do hábitat, devido ao relevo plano da região (ausência de pequenos riachos e hábitats rochosos), podem contribuir para a predominância de espécies com esse modo reprodutivo. Os resultados do presente estudo corroboram apenas em parte aqueles encontrados por PRADO et al. (2005), uma vez que, a área estudada não apresenta sazonalidade quanto à precipitação. Desta forma, a baixa diversificação dos modos reprodutivos registrada no presente estudo e o predomínio de espécies com o modo reprodutivo 1 parecem estar mais relacionados à homogeneidade da área estudada, já que em áreas altamente heterogêneas, como da Floresta Atlântica (e.g., com presença de costões rochosos, riachos de montanha, bromélias, serapilheira úmida), apresentam maior diversidade de modos reprodutivos (HADDAD \& PRADO, 2005).

Segundo Inger \& Cowell (1977), ambientes abertos tendem a ser menos previsíveis e estáveis em relação às condições climáticas se comparados a ambientes florestais. Embora a área aqui estudada não apresente sazonalidade quanto à precipitação, a maioria das espécies utiliza corpos d'água imprevisíveis para reprodução (banhados, açudes/poças) localizados em ambientes abertos. Isso poderia contribuir para a maior ocorrência de espécies com modo reprodutivo 1, seguido dos modos 11 e 30 .

A grande proporção de espécies que desova em ninho de espuma (modos 11 e 30 ) registrada no presente

Tabela II. Hábitats e microhábitats utilizados pelos anuros em uma área de Pampa, Santa Maria, RS, entre novembro de 2001 e outubro de 2002 .

\begin{tabular}{|c|c|c|c|c|c|c|c|c|c|c|c|}
\hline \multirow[b]{2}{*}{ Espécies } & \multicolumn{3}{|c|}{ Sítio de vocalização } & \multicolumn{3}{|c|}{ Tipo de corpo d’água } & \multicolumn{5}{|c|}{ Tipo de matriz } \\
\hline & na água & $\begin{array}{c}\text { sobre } \\
\text { vegetação }\end{array}$ & no solo & banhado & córrego & $\begin{array}{l}\text { açude/ } \\
\text { poça }\end{array}$ & $\begin{array}{l}\text { área } \\
\text { urbana }\end{array}$ & lavoura & campo & capoeira & mata \\
\hline Chaunus ictericus & 1 & 0 & 0 & 1 & 1 & 1 & 1 & 1 & 1 & 1 & 0 \\
\hline Dendropsophus minutus & 0 & 1 & 1 & 1 & 1 & 1 & 1 & 1 & 1 & 1 & 1 \\
\hline Dendropsophus sanborni & 0 & 1 & 0 & 1 & 1 & 1 & 1 & 1 & 1 & 1 & 1 \\
\hline Elachistocleis bicolor & 1 & 0 & 0 & 1 & 0 & 1 & 1 & 1 & 1 & 0 & 1 \\
\hline Hypsiboas albopunctatus & 0 & 1 & 1 & 1 & 1 & 0 & 1 & 0 & 1 & 0 & 0 \\
\hline Hypsiboas pulchellus & 1 & 1 & 1 & 1 & 1 & 1 & 0 & 1 & 1 & 1 & 0 \\
\hline Leptodactylus fuscus & 0 & 0 & 1 & 1 & 1 & 1 & 1 & 1 & 1 & 1 & 0 \\
\hline Leptodactylus gracilis & 0 & 0 & 1 & 1 & 0 & 1 & 1 & 1 & 1 & 1 & 1 \\
\hline Leptodactylus latinasus & 0 & 0 & 1 & 1 & 0 & 1 & 0 & 0 & 1 & 0 & 0 \\
\hline Leptodactylus mystacinus & 0 & 0 & 1 & 1 & 0 & 1 & 0 & 1 & 1 & 0 & 0 \\
\hline Leptodactylus cf. ocellatus & 1 & 0 & 1 & 1 & 0 & 1 & 1 & 1 & 1 & 1 & 0 \\
\hline Lithobates catesbeianus & 1 & 0 & 1 & 1 & 1 & 1 & 1 & 1 & 1 & 1 & 1 \\
\hline Odontophrynus americanus & 1 & 0 & 0 & 1 & 0 & 0 & 0 & 1 & 0 & 0 & 0 \\
\hline Physalaemus biligonigerus & 1 & 0 & 0 & 1 & 0 & 1 & 1 & 1 & 1 & 1 & 0 \\
\hline Physalaemus cuvieri & 1 & 0 & 0 & 1 & 0 & 1 & 1 & 1 & 1 & 1 & 0 \\
\hline Physalaemus henselii & 1 & 0 & 0 & 1 & 0 & 0 & 0 & 0 & 1 & 0 & 0 \\
\hline Physalaemus riograndensis & 1 & 0 & 0 & 1 & 0 & 1 & 1 & 1 & 1 & 0 & 0 \\
\hline Pseudis minuta & 1 & 0 & 0 & 0 & 0 & 1 & 0 & 1 & 1 & 0 & 0 \\
\hline Pseudopaludicola falcipes & 1 & 0 & 1 & 1 & 1 & 1 & 1 & 1 & 1 & 0 & 0 \\
\hline Scinax berthae & 0 & 1 & 0 & 1 & 0 & 1 & 0 & 1 & 1 & 1 & 0 \\
\hline Scinax fuscovarius & 0 & 1 & 1 & 1 & 1 & 1 & 1 & 1 & 1 & 1 & 1 \\
\hline Scinax granulatus & 0 & 1 & 1 & 1 & 0 & 0 & 0 & 0 & 1 & 0 & 0 \\
\hline Scinax nasicus & 0 & 1 & 0 & 1 & 0 & 1 & 1 & 1 & 0 & 1 & 1 \\
\hline Scinax squalirostris & 0 & 1 & 0 & 1 & 1 & 1 & 1 & 1 & 1 & 1 & 0 \\
\hline
\end{tabular}


estudo também parece estar relacionada às características ambientais. Inúmeros autores têm sugerido funções para a construção de ninho de espuma pelos anuros, como proteção contra dessecação dos ovos (Downie, 1988), prover temperaturas adequadas para o desenvolvimento de ovos e embriões (DobKin \& GETTINGER, 1985), refúgio contra predadores (DownIE, 1990) e suprimento adequado de oxigênio para os ovos (SEYMour \& Loveridge, 1994). HöDL (1990) observou, em estudo sobre a diversidade de modos reprodutivos de espécies amazônicas, que as espécies de áreas abertas ou antropicamente alteradas possuem desovas aquáticas ou em ninho de espuma e que, espécies que desovam na vegetação acima dos corpos d'água seriam restritas a formações florestais. B.S.V. Pimenta (com. pes.) observou que a abundância de indivíduos de cada modo reprodutivo mostrou-se altamente dependente do tipo de ambiente, indicando forte relação entre ambientes abertos e modos em que os ovos são colocados diretamente na água ou que envolvem ninho de espuma em taxocenoses de anuros. Segundo Duellman \& Trueb (1986), a diversidade de modos reprodutivos é mais um reflexo das características do ambiente onde os anuros vivem do que de relações filogenéticas de famílias e altas categorias. Desta forma, o padrão encontrado no presente estudo sugere que em áreas subtropicais onde a sazonalidade climática é regida pela temperatura ao invés das chuvas, a homogeneidade do hábitat talvez seja o fator mais importante para explicar a predominância de espécies que desovam diretamente na água ou em ninho de espuma.

O padrão de distribuição da abundância das espécies apresentou melhor ajuste aos modelos Broken Stick e Log-normal ( $p>0,05$; Fig. 2), caracterizados pela homogeneidade na distribuição da abundância das espécies, do que aos modelos Série Geométrica e Logarítmica ( $\mathrm{p}<0,05)$, onde ocorre um pequeno número de espécies abundantes e grande proporção de espécies raras. O modelo Broken Stick foi proposto para comunidades de aves territoriais e outras comunidades onde a competição interespecífica exclui a possibilidade de sobreposição de nicho (MAGURRAN, 1988), uma predição contrária ao observado em comunidades de anuros de áreas abertas, onde geralmente as espécies apresentam forte sobreposição no uso de recursos espaciais e/ou temporais (e.g., CARDoso et al., 1989; Pombal, 1997; Rossa-Feres \& JiM, 2001; VAsCONCELOS \& RossA-Feres, 2005). A maior eqüitabilidade na abundância, predita pelo modelo Log-normal, é geralmente encontrada quando muitos fatores governam a ecologia de uma comunidade e tem sido registrado na maioria dos estudos de comunidades (MAGURRAN, 1988).

Sazonalidade. O período de menor número de espécies com machos em atividade de vocalização foi registrado de abril a julho ( $6 \pm 2,4$ espécies), enquanto que o período de maior número de espécies com machos vocalizando ocorreu de agosto a março (13,9 $\pm 2,9$ espécies) $(\mathrm{U}=2,63 ; \mathrm{p}<0,05)$ (Tab. I). Houve diferença marginalmente significativa $(U=1,95 ; p=0,051)$ entre esses dois períodos quanto à temperatura média mínima (abril-julho: $12,8 \pm 3,2^{\circ} \mathrm{C}$; agosto-março: $16,8 \pm 3,7^{\circ} \mathrm{C}$ ) e significativa $(\mathrm{U}=2,04 ; \mathrm{p}<0,05)$ quanto à temperatura média máxima (abril-julho: $21,9 \pm 2,9^{\circ} \mathrm{C}$; agosto-março: $\left.27,2 \pm 3,9^{\circ} \mathrm{C}\right)$, mas não houve diferença quanto à precipitação acumulada $(\mathrm{U}=16 ; \mathrm{p}>0,05)$ (abril-julho:

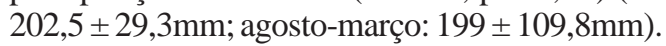

A análise de agrupamento evidenciou a formação de três grupos de espécies com forte sobreposição na temporada de vocalização dos machos (Fig. 3): 1) composto pelas espécies cujos machos vocalizaram durante o inverno e primavera; 2) composto pelas espécies cujos machos vocalizaram preponderantemente durante o período mais quente do ano (primavera e verão) (Tab. I e Fig. 4) e 3) composto pelas espécies cujos machos vocalizaram ao longo de quase todo o ano, inclusive durante o período mais frio (Tab. I e Fig. 4).

Sete espécies não foram associadas a nenhum agrupamento (Fig. 3). Machos de Odontophrynus americanus (Duméril \& Bibron, 1841) e de Physalaemus henselii apresentaram atividade de vocalização restrita a alguns meses de inverno (Tab. I). Machos de Leptodactylus latinasus vocalizaram em janeiro e outubro, machos de Physalaemus biligonigerus (Cope, 1861) em novembro e janeiro e os de $S$. granulatus vocalizaram em agosto e setembro. Machos de Hypsiboas albopunctatus vocalizaram apenas em fevereiro, enquanto os de Scinax nasicus vocalizaram em fevereiro e outubro. A temporada de vocalização dos machos foi possivelmente subestimada para as cinco últimas espécies devido à baixa abundância com que estes ocorreram na área estudada, já que em outros locais próximos, onde observamos grandes agregações de machos (T.G.S. obs. pes.), a temporada de vocalização foi mais ampla e tipicamente associada ao período de primavera e verão. Desta forma, a partilha temporal dos recursos ligados à reprodução parece ter importância secundária no presente estudo, pois houve grande sobreposição na temporada de vocalização durante o período da primavera de verão, quando machos da maioria das espécies vocalizaram.

Todas as variáveis climatológicas, bem como a riqueza de espécies e a abundância de machos em atividade de vocalização, apresentaram correlação com a matriz-hipótese de coesão temporal (Tab. III). Este resultado evidenciou a existência de uma forte estrutura temporal nas variáveis climatológicas e nos dados bióticos, sendo necessária sua retirada para poder identificar se e onde realmente ocorreram correlações e o quanto dessa estrutura foi apenas temporalidade. Após a retirada do efeito da coesão temporal, foram registradas correlações positivas fracas da riqueza de espécies com a precipitação mensal acumulada e da abundância com a temperatura média máxima (Tab.III).

A atividade reprodutiva da maioria das espécies de anuros, em ambientes sazonais, não ocorre ao longo do ano, o que estabelece uma sazonalidade reprodutiva que é condicionada, principalmente, pelas chuvas e temperatura (CARDOSO \& MARTINS, 1987). Correlações da temperatura e/ou precipitação com a riqueza e a abundância de machos em atividade de vocalização têm sido encontradas para comunidades estudadas no sudeste (e.g., Bertoluci, 1998; Bernarde \& KoKubum, 1999; Bertoluci \& Rodrigues, 2002a; Toledo et al., 2003; VAsconcelos \& Rossa-Feres, 2005) e sul (e.g., Bernarde \& Machado, 2001; Conte \& Machado, 2005; Conte \& 
Rossa-FEREs, 2006) do país, sem que tenha sido testada a existência de coesão temporal na estrutura dos dados, o que limita comparações com os resultados aqui encontrados.

A fraca correlação da precipitação com os dados bióticos era esperada, já que na área estudada, as chuvas são bem distribuídas ao longo do ano. Entretanto, a ausência de correlação das temperaturas com a riqueza de espécies em atividade de vocalização não corroborou a diferença registrada na riqueza de espécies entre o período mais quente e mais frio do ano e os resultados

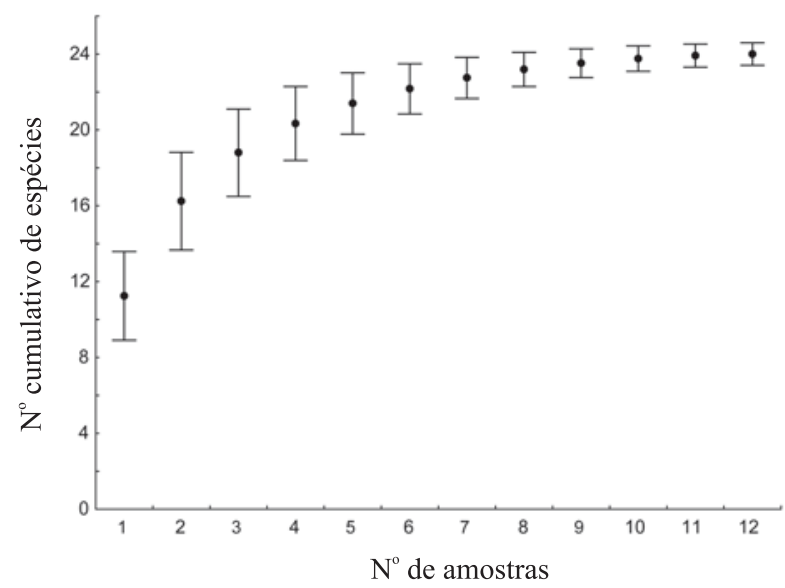

Fig. 1. Curva cumulativa para 24 espécies de anuros registradas em uma área de Pampa, Santa Maria, RS, entre novembro de 2001 e outubro de 2002. Os pontos expressam a curva cumulativa média, gerada por 100 adições aleatórias das amostras, e as barras verticais indicam a variação possível em torno da curva média (intervalo de confiança de $95 \%)$.

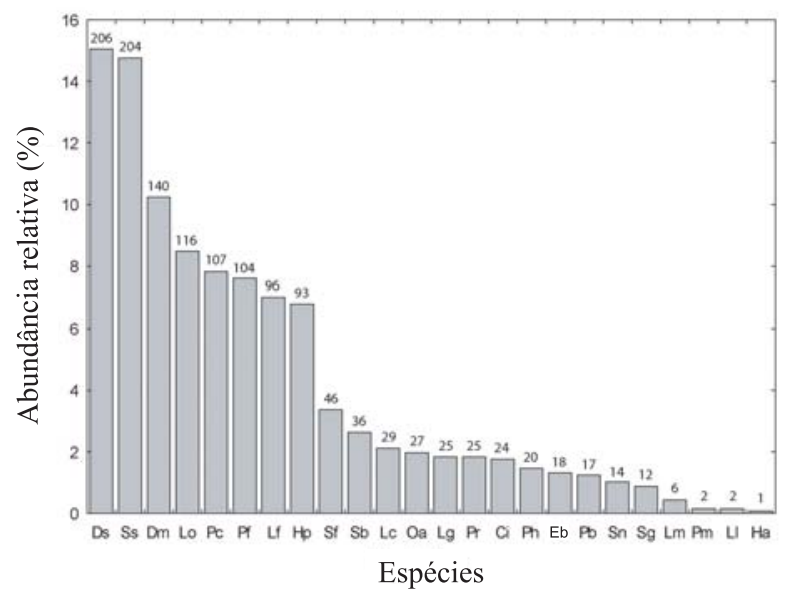

Fig. 2. Curva do componente dominância para 24 espécies de anuros registradas em uma área de Pampa, Santa Maria, RS, entre novembro de 2001 e outubro de 2002, com ajuste aos modelos Broken Stick e Log-normal $(\mathrm{p}>0,05)$. As barras representam a abundância relativa das espécies (\%), enquanto os valores em seus topos representam a abundância absoluta. Espécies: Chaunus ictericus (Ci), Dendropsophus minutus (Dm), D. sanborni (Ds), Elachistocleis bicolor (Eb), Hypsiboas albopunctatus (Ha), H. pulchellus (Hp), Leptodactylus fuscus (Lf), L. gracilis (Lg), L. latinasus (Ll), L. mystacinus (Lm), L. cf. ocellatus (Lo), Lithobates catesbeianus (Lc), Odontophrynus americanus (Oa), Physalaemus cuvieri $(\mathrm{Pc})$, P. biligonigerus $(\mathrm{Pb})$, P. henselii $(\mathrm{Ph})$, P. riograndensis (Pr), Pseudis minuta (Pm), Pseudopaludicola falcipes (Pf), Scinax berthae (Sb), S. fuscovarius (Sf), S. granulatus $(\mathrm{Sg}), S$. squalirostris (Ss) e $S$. nasicus (Sn). obtidos na análise de agrupamento. As correlações obtidas indicaram que na área estudada a temperatura parece atuar mais sobre a abundância de machos em atividade de vocalização e a precipitação sobre a riqueza, apesar da riqueza de espécies ser significativamente maior durante o período mais quente do ano. Um fator que pode ter mascarado as correlações da temperatura com a riqueza e abundância de espécies foi o comportamento oportunista de algumas delas [e.g., Dendropsophus minutus (Peters, 1872), D. sanborni (Schmidt, 1944), Pseudopaludicola falcipes (Hensel, 1867) e Pseudis

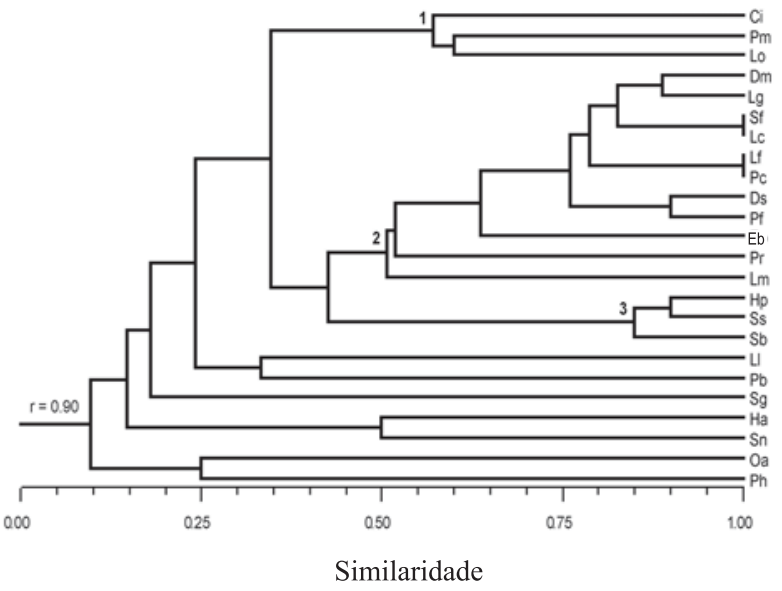

Fig. 3. Similaridade na ocorrência temporal dos machos de 24 espécies de anuros registradas em uma área de Pampa, Santa Maria, RS, entre novembro de 2001 e outubro de 2002. (r, Coeficiente de Correlação Cofenética). Espécies: Chaunus ictericus (Ci), Dendropsophus minutus (Dm), D. sanborni (Ds), Elachistocleis bicolor (Eb), Hypsiboas albopunctatus (Ha), H. pulchellus (Hp), Leptodactylus fuscus (Lf), L. gracilis (Lg), L. latinasus (L1), L. mystacinus (Lm), L. cf. ocellatus (Lo), Lithobates catesbeianus (Lc), Odontophrynus americanus (Oa), Physalaemus cuvieri $(\mathrm{Pc})$, $P$. biligonigerus $(\mathrm{Pb})$, P. henselii $(\mathrm{Ph})$, P. riograndensis $(\mathrm{Pr})$, Pseudis minuta (Pm), Pseudopaludicola falcipes (Pf), Scinax berthae (Sb), S. fuscovarius (Sf), S. granulatus $(\mathrm{Sg}), S$. squalirostris (Ss) e $S$. nasicus (Sn). Agrupamentos de espécies com similaridade superior a 50\%: 1) espécies cujos machos vocalizaram durante o inverno e a primavera; 2) espécies cujos machos vocalizaram preponderantemente durante o período mais quente do ano e 3) espécies cujos machos vocalizaram ao longo de quase todo o ano, inclusive durante o período mais frio.

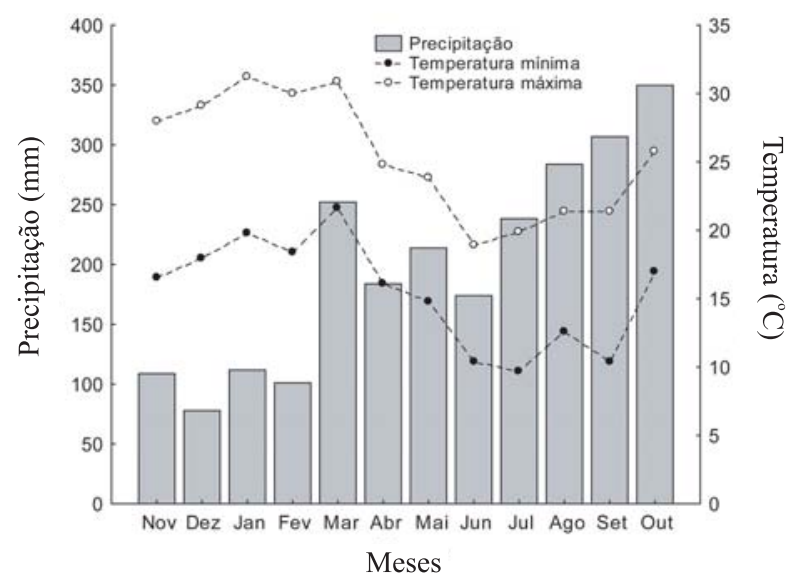

Fig. 4. Precipitação mensal acumulada ( $\mathrm{mm}$; barras) e média mensal das temperaturas mínimas e máximas $\left({ }^{\circ} \mathrm{C}\right.$; linhas) registradas de novembro de 2001 a outubro de 2002 em uma área de Pampa, Santa Maria, RS. 
Tabela III. Correlações de Mantel (r) das matrizes de riqueza (CJ) e abundância $(\mathrm{CH})$ de espécies de anuros, temperatura média mínima (Tmin), temperatura média máxima (Tmax) e de precipitação mensal acumulada $(\mathrm{Pa})$ com a matriz-hipótese de coesão temporal (CT) em uma área de Pampa, Santa Maria, RS, estudada entre novembro de 2001 e outubro de 2002. (- CT) indica a exclusão do efeito da CT sobre as demais matrizes correlacionadas, utilizando o teste de Mantel Parcial.

\begin{tabular}{lcl}
\hline Matrizes & $\mathrm{r}$ & $\mathrm{p}$ \\
\hline CJ vs. CT & 0,50 & 0,0002 \\
CH vs. CT & 0,61 & 0,0002 \\
Tmin vs. CT & 0,43 & 0,0020 \\
Tmax vs. CT & 0,56 & 0,0002 \\
Pa vs. CT & 0,30 & 0,0300 \\
CJ vs. Tmin (- CT) & 0,02 & 0,42 \\
CJ vs. Tmax (- CT) & 0,18 & 0,11 \\
CJ vs. Pa (- CT) & 0,25 & 0,04 \\
CH vs. Tmin (- CT) & 0,08 & 0,23 \\
CH vs. Tmax (- CT) & 0,24 & 0,03 \\
CH vs. Pa (- CT) & 0,09 & 0,21 \\
\hline
\end{tabular}

minuta] cujos machos vocalizaram esporadicamente durante dias mais quentes no inverno. Desta forma, os resultados obtidos revelaram que as variáveis climatológicas testadas explicaram muito pouco da ocorrência sazonal das espécies. Segundo Dodd \& Charest (1988), existe um grande número de variáveis que influencia a distribuição sazonal das espécies, tais como hidroperíodo, temperaturas máximas e mínimas, quantidade e regularidade das chuvas. Ainda segundo esses autores, estas variáveis estão sujeitas a variações diárias, mensais e anuais e sua interação seria a responsável pela variação dos ciclos de atividade das espécies. Nesse estudo, foram verificadas apenas correlações da temperatura e precipitação sobre a riqueza e abundância das espécies. Em estudos futuros sobre a atividade reprodutiva de anuros, a influência de outras variáveis ambientais com variação marcadamente sazonal (e.g., fotoperíodo) merece ser testada na região.

Agradecimentos. Os autores agradecem aos dois revisores anônimos pelas valiosas críticas e sugestões, ao Dr. David J. Rosen pela revisão do Abstract, às colegas do Laboratório de Herpetologia da Universidade Federal de Santa Maria, biólogas Gisele Winck e Camila Both, pelo auxílio nas atividades de campo e aos vigilantes da UFSM pela segurança e solicitude prestada.

\section{REFERÊNCIAS BIBLIOGRÁFICAS}

Achaval, F. \& Olmos, A. 2003. Anfibios y Reptiles del Uruguay. Montevideo, Graphis Impresora. $136 \mathrm{p}$.

Alvarez, B. B.; Aguirre, R. H.; Céspedez, J. A.; Hernando, A. B. \& Tedesco, M. E. 2002. Atlas de anfíbios y reptiles de lãs províncias de Corrientes, Chaco y Formosa (Argentina). Corrientes, Universidad Nacional del Nordeste. 160p.

Ayres, M.; Ayres Jr., M.; Ayres, D. L. \& SAntos, A. S. 2003. BioEstat 3.0. Aplicações estatísticas nas áreas das ciências biológicas e médicas. Belém, Sociedade Civil de Mamirauá. 291p.

Bencke, G. A. 2003. Apresentação. In: Fontana, C. S.; Bencke, G. A. \& ReIS, R. E. orgs. Livro vermelho da fauna ameaçada de extinção no Rio Grande do Sul. Porto Alegre, EDIPUCRS. p.14-21.

Bernarde, P. S. \& Anjos, L. 1999. Distribuição espacial e temporal da anurofauna no Parque Estadual Mata dos Godoy, Londrina, Paraná, Brasil (AMPHIBIA, ANURA). Comunicações do Museu de Ciências e Tecnologia PUCRS, Série Zoologia,
12:111-140.

Bernarde, P. S. \& Konubum, M. N. C. 1999. Anurofauna do Município de Guararapes, estado de São Paulo, Brasil (Amphibia, Anura). Acta Biologia Leopoldensia 21:89-97.

Bernarde, P. S. \& Machado, R. A. 2001. Riqueza de espécies, ambientes de reprodução e temporada de vocalização da anurofauna em Três Barras do Paraná, Brasil (Amphibia: Anura). Cuadernos de Herpetología 14(2):93-104.

Bertoluci, J. 1998. Annual patterns of breeding activity in Atlantic Rainforest anurans. Journal of Herpetology 32(4):607-611.

Bertoluci, J. \& Rodrigues, M. T. 2002a. Seasonal patterns of breeding activity of Atlantic Rainforest anurans at Boracéia, Southeastern Brazil. Amphibia-Reptilia 23:161-167.

2002b. Utilização de hábitats reprodutivos e micro-hábitats de vocalização em uma taxocenose de anuros (AMPHIBIA) DA Mata Atlântica do Sudeste do Brasil. Papéis Avulsos de Zoologia 42(11):287-297.

Braun, C. B. \& Braun, C. A. S. 1974. Fauna da Fronteira BrasilUruguai, lista dos anfíbios dos Departamentos de Artigas, Rivera e Cerro Largo. Iheringia, Série Zoologia, (45):34-39. 1976. Contribuição ao estudo da fauna anfibiológica de região metropolitana (Grande Porto Alegre), Rio Grande do Sul, Brasil. Comunicações do Museu de Ciências da PUCRS (10): 1-16.

1980. lista prévia dos anfíbios do Estado do Rio Grande do Sul, Brasil. Iheringia, Série Zoologia, (56):121-146.

Cardoso, A. J. \& Martins, J. E. 1987. Diversidade de anuros durante o turno de vocalizações em comunidade neotropical. Papéis Avulsos Zoologia 36:279-285.

Cardoso, A. J.; Andrade, G. V.\& Haddad, C. F. B. 1989. Distribuição espacial em comunidades de anfíbios (Anura) no sudeste do Brasil. Revista Brasileira de Biologia 49(1):241-249.

Cechin, S. Z.; Santos, T. G. Dos; Kopp, K. A.; Spies, M. R. \& Trevisan, R. 2002. Scinax nasica. Herpetological Review 33(3): 220 .

CeI, J. M. 1980. Amphibians of Argentina. Italian Journal of Zoology 2:1-609.

Céspedez, J. A.; Aguirre, R. H. \& Alvarez, B. B. 1995. Composicion y distribuicion de la anfibiofauna de la província de Corrientes (Argentina). Facena 11:25-49.

Colwell, R. K. 2004. EstimateS 7.0: Statistical estimation of species richness and shared species from samples. User's guide and application published at: http:// viceroy.eeb.unconn.edu/estimates. Acesso em: 10.06.2005.

Conte, C. E. \& Machado, R. A. 2005. Riqueza de espécies e distribuição espacial e temporal em comunidade de anfíbios anuros (Amphibia, Anura) em uma localidade do Município de Tijucas do Sul, Paraná, Brasil. Revista Brasileira de Zoologia 22(4):940-948.

Conte, C. E. \& Rossa-Feres, D. C. 2006. Diversidade e ocorrência temporal da anurofauna (Amphibia, Anura) em São José dos Pinhais, Paraná, Brasil. Revista Brasileira de Zoologia 23(1): 162-175.

CRumP, M. L. 1971. Quantitative analysis of the ecological distribution of a tropical herpetofauna. Occasional Papers of the Museum of Natural History 3:1-62

1974. Reprodutive strategies in a tropical anuran community. Miscellaneous publications Natural History University Kansas 61:1-68.

Di-Bernardo, M. \& Kwet, A. 2002. Efeitos da contaminação de águas superficiais associadas a atividades de extração e processamento de carvão sobre anfíbios. In: Teixeira, E. C. \& Pires, M. J. R. eds. Meio ambiente e carvão - Impactos da exploração e utilização. Porto Alegre, FEPAM. p.413-422.

Di-Bernardo, M.; Oliveira, R. B.; Pontes, G. M. F.; Melchiors, J.; Solé, M. \& Kwet, A. 2004. Anfíbios anuros da região de extração e processamento de carvão de Candiota, RS, Brasil. In: Teixeira, E. C. \& Pires, M. J. R. eds. Estudos ambientais em Candiota: carvão e seus impactos. Porto Alegre, FEPAM. p.163-175.

Dobkin, D. S. \& GetTinger, R. D. 1985. Thermal aspects of anuran foam nests. Journal of Herpetology 19:271-275.

DodD, K. D. \& B. G. Charest. 1988. The herpetofaunal community of temporary ponds in north Florida sandhills: species composition, temporal use, and management implications. In: Szaro, R. C.; Severson, K. E. \& Patton, D. R. orgs. 
Management of amphibians, reptiles, and small mammals in North America. Proceedings of the Symposium. Flagstaff, USDA Forest Service. p.87-97.

Downie, J. R. 1988. Functions of the foam in the foam-nesting leptodactylid Physalaemus pustulosus. Herpetological Journal 1:302-307.

. 1990. Functions of the foam in a foam-nesting leptodactylids: anti-predator effects of Physalaeumus pustulosus foam. Herpetological Journal 1:501-503.

Duellman, W. E. \& Trueb, L. 1986. Biology of amphibians. New York, McGraw-Hill Book Company. 670p.

Eterovick, P. C.; Carnaval, A. C. O. Q.; Borges-Nojosa, D. M.; Silvano, D. L.; Segalla, M. V.; Sazima, I. 2005. Amphibian declines in Brazil: an overview. Biotropica 37(2):166-179.

Garcia, P. C. DE A. \& Vinciprova, G. 1999. Range extensions of some anuran species for Santa Catarina and Rio Grande do Sul States, Brazil. Herpetological Review 29(2):117-118. . 2003. Anfíbios. In: Fontana, C. S.; Bencke, G. A. \& Reis, R. E orgs. Livro vermelho da fauna ameaçada de extinção no Rio Grande do Sul. Porto Alegre, EDIPUCRS. p.147-164.

Gayer, S. M. P.; Krause, L. \& Gomes, N. 1988. Lista preliminar dos anfíbios da Estação Ecológica do Taim, Rio Grande do Sul, Brasil. Revista Brasileira de Zoologia 5(3):419-425.

Grando, J. V.; Gonçalves, F. A. \& Zanella, N. 2004. Composição e distribuição estacional dos anuros de um remanescente de floresta nativa em área urbana no município de Passo Fundo, RS. Acta Biológica Leopoldensia 26(1):93-100.

Haddad, C. F. B. \& Abe, A. 2000. Anfíbios e Répteis. In Workshop Mata Atlântica e Campos Sulinos. Conservation International do Brasil, Fundação Biodiversitas, Fundação SOS Mata Atlântica, Instituto de Pesquisas Ecológicas, Secretarias de Meio Ambiente de São Paulo e Minas Gerais. Disponível em: <http://www.conservation.org.br/ publicacoes/files/Sumario.pdf $>$.

Haddad, C. F. B. \& Prado, C. P. A. 2005. Reproductive modes in frogs and their unexpected diversity in the Atlantic Forest of Brazil. BioScience 55(3):207-217.

Heyer, W. R. \& Bellin, M. S. 1973. Ecological notes on five sympatric Leptodactylus (Amphibia, Leptodactylidae) from Ecuador. Herpetologica 29(1):66-72.

HöDL, W. 1977. Call difference and vocalizing sites segregation in anuran species from Central Amazonian floating meadows. Oecologia 28:351-363.

1990. Reproductive diversity in Amazonian lowland frogs. In: Hanke, W. ed. Biology and Physiology of Amphibians. New York, Gustav Fischer Verlag. p.62-73.

IBGE. 2004. Mapa de Biomas do Brasil. Primeira aproximação. Disponível em: <http://www2.ibge.gov.br/ download/mapas_murais/biomas_pdf.zip>. Acesso em: 12.06.2006.

Inger, R. F. \& Colwell, R. K. 1977. Organization of contiguous communities of amphibians and reptiles in Thailand. Ecological Monographs 47:229-253.

Kopp, K. A.; SAntos, T. G. Dos; Spies, M. R.; Trevisan, R. \& Cechin, S. Z. 2002. Hyla albopunctata. Herpetological Review 33(3): 222 .

Kopp, K. \& Eterovick, P. C. 2006. Factors influencing spatial and temporal structure of frog assemblages at ponds in southeastern Brazil. Journal of Natural History 40:1813-1830.

Krebs, C. J. 1999. Ecological Methodology. Menlo Park, Addison Waley Longman Inc. 620p.

Kwet, A. 2000. The genus Pseudis (Anura: Pseudidade) in Rio Grande do Sul, southern Brazil, with description of a new species. Amphibia-Reptilia 21:39-55.

2001. Frösche im brasilianischen AraukarienwaldAnurengemeinschaft des Araukarienwaldes von Rio Grande do Sul: Diversität, Reproduktion und Ressourcenaufteilung. Tübingen, Natur und Tier-Verlag. 192 p.

Kwet, A. \& Di-Bernardo, M. 1998. Elachistocleis erythrogaster, a new microhylid species from Rio Grande do Sul, Brazil. Studies of Neotropical Fauna \& Environment 33:7-18.

1999. Pró-Mata: Anfíbios-Amphibien-Amphibians. Porto Alegre, EDIPUCRS. 107p.

Kwet, A. \& Faivovich, J. 2001. Proceratophrys bigibbosa species group (Anura: Leptodactylidae), with description of a new species. Copeia 2001:203-215.
Langone, J. A. 1994. Ranas y sapos del Uruguay. Montevideo, Museo Damaso Antônio Larraña. 123p.

Leite, P. \& Klein, R. M. 1990. Vegetação. In: IBGE. Geografia do Brasil: região sul. Rio de janeiro, Instituto Brasileiro de Geografia e Estatística. p.113-150.

Loebmann, D. \& Vieira, J. P. 2005. Relação dos anfíbios do Parque Nacional da Lagoa do Peixe, Rio Grande do Sul, Brasil. Revista Brasileira de Zoologia 22(2):339-341.

Magurran, A. E. 1988. Ecological diversity and its measurement. Princeton, Princeton University. 192p.

Maneyro, R. \& Langone, J. A. 2001. Categorización de los anfíbios del Uruguay. Cuadernos de Herpetología 15(2):107-118.

Manly, B. F. G. 1994. A Primer of Multivariate Statistics. London, Chapmam \& Hall. 179p.

Mantovani, W. \& Silva, S. M. 2002. Vegetação e Flora. In: MMA. Biodiversidade Brasileira: Mata Atlântica e Campos Sulinos. Brasília, Ministério do Meio Ambiente. p.219-225.

Martins, M. B.; Di-Bernardo, M.; Vinciprova, G.; Measey, G. J. 2002. Geographic distribution. Rana catesbeiana. Herpetological Review 33(4):319.

MMA. 2002. Biodiversidade Brasileira - avaliação e identificação de áreas e ações prioritárias para conservação, utilização sustentável e repartição de benefícios da biodiversidade brasileira. Brasília, MMA/SBF. 404p.

Pereira, P. R. B.; Netto, L. R. G.; Borin, C. J. A. \& Sartori, M. G. B. 1989. Contribuição à geografia física do município de Santa Maria: unidades de paisagem. Geografia - Ensino \& Pesquisa 3:37-68.

Pombal JR., J. P. 1997. Distribuição espacial e temporal de anuros (Amphibia) em uma poça permanente na Serra de Paranapiacaba, sudeste do Brasil. Revista Brasileira de Biologia 57(4):583-594.

Porto, M. L. 2002. Os Campos Sulinos: sustentabilidade e manejo. Ciência \& Ambiente 24(4):119-138.

Prado, C. A.; Uetanabaro, M. \& Haddad, C. F. B. 2005. Breeding activity patterns, reproductive modes, and habitat use by anurans (Amphibia) in a seasonal environment in the Pantanal, Brazil. Amphibia-Reptilia 26:211-221.

Risser, P. G. 1997. Diversidade em e entre prados. In: Wilson, E. O. org. Biodiversidade. Rio de Janeiro, Nova Fronteira. p. 224-229.

Rohlf, F. J. 2000. NTSYS 2.1: Numerical Taxonomic and Multivariate Analysis System. New York, Exeter Software.

Romesburg, H. C. 1984. Cluster Analysis for researchers. Malabar, Robert E. Krieger Publishing Company. 334p.

Rossa-Feres, D. C. \& Jim, J. 1994. Distribuição sazonal em comunidades de anfíbios anuros na região de Botucatu, São Paulo. Revista Brasileira de Biologia 54(2):323-334.

. 2001. Similaridade no sítio de vocalização em uma comunidade de anfíbios anuros na região noroeste do estado de São Paulo, Brasil. Revista Brasileira de Zoologia 18(2):439-454.

Santos, T. G.; Kopp, K. A.; Spies, M. R.; Trevisan, R. \& Cechin, S. Z. 2005. Répteis do Campus da Universidade Federal de Santa Maria, RS, Brasil. Biota Neotropica 5(1): <http:// www.biotaneotropica.org.br/v $5 \mathrm{n} 1 / \mathrm{pt} / \mathrm{abstract}$ ? inventory+ BN02705012005>.

Santos, T. G.; Rossa-Feres, D. C. \& Casatti, L. 2007. Diversidade e distribuição espaço-temporal de anuros em região com pronunciada estação seca no sudeste do Brasil. Iheringia, Série Zoologia, 97(1):37-49.

SBH. 2008. Lista de espécies de anfíbios do Brasil. Sociedade Brasileira de Herpetologia (SBH). Disponível em: <http:// www.sbherpetologia.org.br/checklist/anfibios.htm>. Acesso em 01.06.2008.

ScotT JR., N. J. \& Woodward, B. D. 1994. Surveys at breeding sites. In: Heyer, W. R.; Donnelly, M. A.; McDiarmid, R. W.; Hayek, L. A. C. \& Foster, M. S. orgs. Measuring and Monitoring Biological Diversity - Standard Methods for Amphibians. Washington, Smithsonian Institution Press. p. $84-92$

Seymour, R. S. \& Loveridge, J. P. 1994. Embryonic and larval respiration in the arboreal foam nests of the African frog Chiromantis xerampelina. Journal of Experimental Biology 197:31-46. 
Silveira-Neto, S.; Nakano, O.; Barbin, D. \& Nova, N. A. V. 1976. Manual de ecologia dos insetos. São Paulo, Editora Agronômica Ceres. 419p.

Smouse, P. E.; Long, J. C. \& Sokal, R. R. 1986. Multiple regression and correlation extensions of the Mantel test of matrix correspondence. Systematic and Zoology 35(4):627-632.

Straneck, R.; Olmero, E. V. \& Carrizo, G. R. 1994. Catalogo de voces de anfibios argentinos. Buenos Aires, L.O.L.A. 127p.

Toledo, L. F.; Zina, J. \& Haddad, C. F. B. 2003. Distribuição Espacial e Temporal de uma Comunidade de anfíbios Anuros do Município de Rio Claro, São Paulo, Brasil. Holos Environment 3(2):136-149.

Vasconcelos, T. da S. \& Rossa-Feres, D. C. 2005. Diversidade, distribuição espacial e temporal de anfíbios anuros (Amphibia, Anura) na região noroeste do estado de São Paulo, Brasil. Biota Neotropica 5(2): <http://www.biotaneotropica.org.br/ v5n2/pt/abstract?article+BN01705022005>.

ZAR, J. H. 1999. Biostatistical Analysis. Upper Saddle River, Prentice Hall. 929p.

Recebido em julho de 2006. Aceito em outubro de 2007. ISSN 0073-4721

Artigo disponível em: www.scielo.br/isz 